
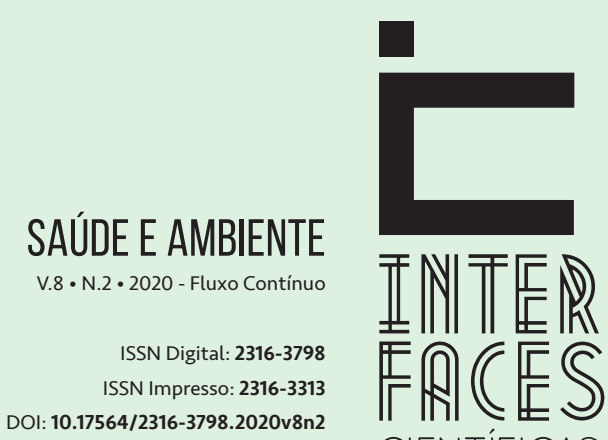

CIENTÍFICAS

\section{AMBIÊNCIA DA SALA DE ESPERA ODONTOLÓGICA E ASPECTOS PSICOSSOCIAIS DE USUÁRIOS DE UNIDADES BÁSICAS DE SAÚDE}

DENTAL WAITING ROOM AMBIENCE AND PSYCHOSOCIAL ASPECTS USERS' OF BASIC HEALTH UNITS

\section{AMBIENTE DE SALA DE ESPERA ODONTOLÓGICA Y ASPECTOS PSICOSOCIALES DE USUARIOS DE UNIDADES BÁSICAS DE SALUD}

Liziane Monique de Souza Cardoso ${ }^{1}$ Jefferson Chaves Moreira ${ }^{2}$

Marlos Cesar Bomfim Cabral ${ }^{3}$ Regiane Cristina do Amaral ${ }^{4}$

\section{RESUMO}

0 medo e a ansiedade podem interferir negativamente no procedimento odontológico, sendo importante o estudo de meios farmacológicos e/ou não farmacológicos de amenizá-los. Dessa forma, objetivou-se conhecer a percepção dos usuários de Unidades Básicas de Saúde (UBS) do município de Ribeirópolis - SE sobre a ambiência da sala de espera odontológica e aspectos psicossociais envolvidos neste âmbito. Coletaram-se dados por meio de entrevista utilizando-se a Dental Anxiety Scale (DAS)e o Perceived Hospital Environment Quality Indicator (PHEQI), além de questões socioeconômicas e educacionais. Foram entrevistados 308 pacientes, em quatro UBSs. Relacionando a DAS com as variáveis socioeconômicas, verifica-se diferença estatisticamente significativa para os itens gênero e idade, sendo que $72.7 \%$ dos avaliados apresentam ansiedade dental. Aplicando o PHEQI, 60\% consideram o ambiente clínico em boa qualidade ou condições. Conclui-se que os usuários consideram a ambiência conveniente às suas condições socioeconômicas, mas vislumbram a necessidade de melhorias. Por fim, reafirma-se que o ambiente da sala de espera é capaz de interferir positiva ou negativamente na ansiedade relativamente à harmonia de sua ambiência.

\section{PALAVRAS-CHAVE}

ansiedade, percepção, saúde pública 


\section{ABSTRACT}

Fear and anxiety may negatively interfere with the dental procedure, it is important to study pharmacological and/or non-pharmacological means of mitigating them. Thus, this study aimed to understand the perception of users of Basic Health Units from Ribeirópolis - SE, about the ambience of the dental waiting room and the psychosocial aspects involved in this area. Data were collected through interviews using the Dental Anxiety Scale (DAS) and Perceived Hospital Environment Quality Indicator (PHEQI), and socioeconomic and educational issues also. Three hundred eight patients were interviewed in four Basic Health Units. Relating the DAS with the socioeconomic variables, there is a statistically significant difference for the items gender and age, of which $72.7 \%$ of the subjects had dental anxiety. Applying the PHEQI, $60 \%$ the parameter must be in good quality or condition. It is concluded that users consider the ambience convenient to their socioeconomic conditions, but aims for improvements. Thus, it is reaffirmed that the waiting room is capable of positively or negatively interfering with the harmony of its ambience.

\section{KEYWORDS}

Anxiety, Perception, Public Health

\section{RESUMEN}

El miedo y la ansiedad pueden interferir negativamente con el procedimiento dental, y es importante estudiar medios farmacológicos y / o no farmacológicos para aliviarlos. Así, el objetivo fue conocer la percepción de los usuarios de las Unidades Básicas de Salud (BHU) en Ribeirópolis - SE sobre el ambiente de la sala de espera dental y los aspectos psicosociales involucrados en esta área. Los datos fueron recolectados a través de entrevistas usando la Escala de ansiedad dental (DAS) y el Indicador de calidad del ambiente hospitalario percibido (PHEQI), así como cuestiones socioeconómicas y educativas. Se entrevistaron 308 pacientes en cuatro UBS. Relacionando el DAS con las variables socioeconómicas, existe una diferencia estadísticamente significativa para los ítems género y edad, y el $72.7 \%$ de los evaluados tienen ansiedad dental. Aplicando el PHEQI, el 60\% considera el entorno clínico en buena calidad o condición. Se concluye que los usuarios consideran que el ambiente es conveniente para sus condiciones socioeconómicas, pero ven la necesidad de mejorar. Finalmente, se reafirma que el ambiente de la sala de espera puede interferir positiva o negativamente con la ansiedad sobre la armonía de su ambiente. 


\section{PALABRAS CLAVE}

Ansiedad, percepción, salud pública

\section{INTRODUCÇÃO}

A Política Nacional de Humanização (PNH) - Humaniza SUS (BRASIL, 2003) efetiva os princípios do SUS no cotidiano das práticas de atenção e gestão, qualificando a saúde pública no Brasil ao incentivar trocas solidárias entre gestores, trabalhadores e usuários. Dentre os métodos de aplicabilidade têm-se o acolhimento e a ambiência que são dispositivos importantes para assegurar ao usuário um melhor atendimento. A ambiência une os conceitos de ambiente e vivência, e tem na área de saúde, a sua importância relacionada ao espaço acolhedor e saudável no qual os usuários do serviço e os trabalhadores tenham seus vínculos sociais facilitados, em que as relações de cuidado sejam humanizadas e resolutivas (BRASIL, 2003).

Entre os sistemas de saúde pública brasileiros, tem-se a Atenção Primária em Saúde (APS), considerada a porta de entrada e responsável pelo fluxo do cidadão na rede (MENDES, 2015), esta permite que a estrutura da Unidade Básica de Saúde (UBS) possa ter ambiência aprazível, fortalecendo a integralidade do acolhimento (GARCIA et al., 2014), bem como reorientando o cuidado à saúde (BRASIL, 2006).

Neste contexto, a sala de espera é o ambiente que costuma propiciar vínculos entre usuários e profissionais (EMMI; PIRES, 2016), pois nela circulam muitos pacientes (REIS et al., 2014) favorecendo que a ambiência deste local integre a humanização do serviço (VALENTE et al., 2015).

Alguns métodos, visando promover redução da ansiedade na sala de espera, são utilizados pelos profissionais que trabalham na APS, tais como brincadeiras, acesso a uma sala de jogos e distribuição de livros para colorir, para pacientes infantis, bem como música, aromaterapia e design de interiores, para os adultos (BIDDISS et al., 2014). Ainda, atividades de educação em saúde, também podem ser uma opção para ambos os grupos, uma vez que, além de multiplicar saberes e propor interação social, atenuam a ansiedade ao humanizar o serviço (REIS et al., 2014).

Assim, uma experiência agradável em uma sala de espera constitui um instrumento para o alívio da ansiedade (RODRIGUES et al., 2015), por consequência, o tempo ocioso do usuário pode ser otimizado (EMMI et al., 2016), lançando mão de tecnologias leves na produção do cuidado (MERHY, 2005).

Em vista disso, o presente estudo teve por objetivo conhecer a percepção dos usuários que frequentam os consultórios odontológicos das UBS em relação à ambiência da sala de espera e seus aspectos psicossociais, no município de Ribeirópolis-SE. 


\section{MÉTODOS}

Trata-se de um estudo transversal, em que foram aplicadas a escala Dental Anxiety Scale(DAS) ou “Escala de Ansiedade Dental" de Corah (CORAH, 1969) e o questionário de fator "conforto físico e espacial" do Perceived Hospital Environment Quality Indicator(PHEQI) ou Indicador de Qualidade do Ambiente Hospitalar Percebido (FORNARA et al., 2006), além de um roteiro de entrevista socioeconômico e educacional.

\subsection{INDICADOR DE QUALIDADE DO AMBIENTE HOSPITALAR PERCEBIDO [PHEQI]}

Estes instrumentos foram aplicados nos meses de setembro a novembro de 2019 após aprovação do projeto pela Secretaria Municipal de Saúde de Ribeirópolis e pelo Comitê de Ética em Pesquisa (CEP) da Universidade Federal de Sergipe (UFS), seguindo as diretrizes e normas regulamentadoras da Resolução n 466/12 do Conselho Nacional de Saúde (CAEE 10268919.9.0000.5546).

Assim usuários de Unidades Básicas de Saúde (UBS) do município de Ribeirópolis-SE, maiores de 18 anos e que frequentavam as UBS para suas consultas, responderam os questionários a fim de se conhecer a ambiência da sala de espera odontológica e aspectos psicossociais.

Foram avaliadas quatro UBS, contudo, a UBS localizada na sede do município teve um número maior de entrevistados, devido a facilidade de acesso, pois as demais se encontram em locais mais remotos e com pouco fluxo de transporte público.

\subsection{CARACTERÍSTICAS DO MUNICÍPIO}

O município de Ribeirópolis (FIGURA 1), o qual se estende por 259,022 km² os quais compreendem 17.173 pessoas, com uma estimativa de 18.528 para 2018. 0 município está localizado no estado de Sergipe, a 75 km da capital Aracaju, e dispõe do melhor Produto Interno Bruto (PIB) da microrregião do Agreste de Carira, tendo renda média mensal de 1,8 salários mínimos e IDH 0,613 (IBGE, 2010).

Figura 1 - Localização do município de Ribeirópolis - SE. Ribeirópolis, 2019
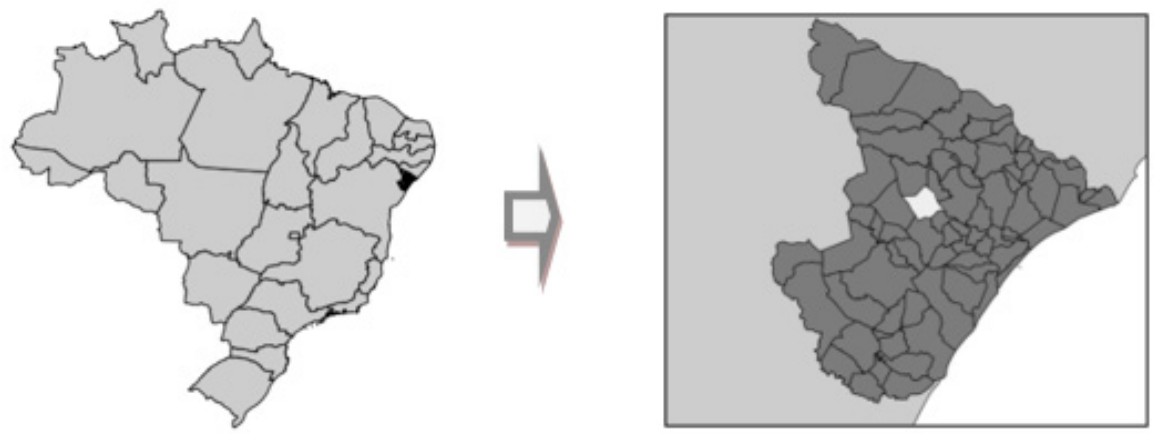

Fonte: Autores 
Em Ribeirópolis, existem doze UBS cadastradas no Sistema de Cadastro Nacional de Estabelecimentos de Saúde (SCNES), sete Equipes de Saúde da Família (ESF) e quatro Equipes de Saúde Bucal (ESB) modalidade I (Cirurgião-Dentista e Auxiliar em Saúde Bucal). Distribuem-se no município cinco consultórios odontológicos, sendo dois na Clínica de Saúde Dr. Dijaume Francisco de Lima, localizada na sede do município, um no Posto de Saúde Eustáquio de Andrade, no Conjunto José Francisco do Nascimento, um no Centro de Saúde Irmã Alice de Jesus Fernandes, Povoado Serra do Machado e um no Posto de Saúde Gevanda do Nascimento, Povoado Riachinho (BRASIL, 2017).

\subsection{AMOSTRA}

Para o cálculo do tamanho amostral foi utilizado amostra aleatória simples sem reposição do indivíduo amostrado na aleatorização, utilizando o programa Microsoft Excel, com base no número de primeiras consultas odontológicas programáticas em proporção à quantidade de usuários adultos do serviço odontológico em Ribeirópolis, de acordo com o eSUS. Assim, para o ano de 2018 foram realizadas 1728 primeiras consultas, somando as 4 UBS, utilizando nível de confiança de 95\% e margem de erro de $5 \%$, o tamanho amostral foi de 325 voluntários.

Assim os usuários foram abordados (aproximadamente 340 pessoas) e perguntado se estes tinham interesse em participar do estudo. Os que desejassem participar assinaram o Termo de Consentimento Livre e Esclarecido (TCLE) e responderam as questões. Foi realizado análise dos questionários e somente analisados os que estavam corretamente preenchidos, desta forma o número final de questionários avaliados foi de 308.

O público-alvo do estudo foram os usuários que aguardavam por atendimento odontológico nas salas de espera das UBS avaliadas e que tinham idade superior a 18 anos. 0 critério de seleção foi estar presente na UBS, ser maior que 18 anos e desejar participar da pesquisa.

\subsection{INSTRUMENTOS}

Antes de iniciar a pesquisa foi realizado um estudo piloto com 20 usuários em uma das UBS, a fim de verificar se os mesmos entenderiam as questões aplicadas, principalmente aquelas que não eram derivadas de questionários validados.

Em função disso, foi utilizado um roteiro de entrevista estruturado com perguntas abertas e fechadas, abordando questões relativas à ansiedade frente ao tratamento odontológico, utilizando-se a DAS (CORAH, 1969) e para avaliar a percepção quanto a ambiência da sala de espera foi utilizado 6 itens do PHEQI (FORNARA et al., 2006).

O DAS consiste em quatro questões de múltipla escolha sobre a subjetividade do paciente frente ao tratamento odontológico em diferentes situações, nela o grau de ansiedade odontológica é classificado por pontuação: sendo até 5 pontos considerado muito pouco ansioso ou nulo, de 6 a 10 levemente ansioso, 11 a 15 moderadamente ansioso e 16 a 20 extremamente ansioso (CORAH, 1969).

O PHEQI, por sua vez, é uma adaptação (FORNARA et al., 2006), do Perceived Residential Environment Quality Indicator (PREQI) (BONAIUTO et al., 2003), feita com o objetivo de criar um instrumento 
que possa ser utilizado para avaliar um ambiente de saúde a partir da percepção objetiva de especialistas, como arquitetos, também da percepção subjetiva de leigos, no caso, usuários, visitantes e funcionários, a fim de comparar ambientes de diferentes graus de humanização. Ele baseia-se em questões sobre os aspectos físico-espaciais e sociofuncionais com respostas em escalas do tipo Likert de 5 pontos para cada item (de "discordo totalmente" a "concordo totalmente").

Cada escala contém um número igual de itens positivos (isto é, indicando a presença de qualidade) e negativos (isto é, indicando a ausência de qualidade), para controlar o conjunto de respostas. Neste trabalho, usamos 6 itens relacionados ao conforto físico e espacial do PHEQI, os quais mostraram-se pertinentes ao nosso objetivo (FORNARA et al., 2006).

Para análise dos dados foram aplicadas análises descritivas e qui-quadrado, utilizando os programas Excel e Bioestat 5.0. Foi ainda realizada a correlação de Pearson entre a DAS e questões do PHEQI, usando o programa SPSS17.0; intervalo de confiança de $95 \%$.

\section{RESULTADOS}

Foram entrevistados 308 pacientes, com uma média de idade de 39 anos, sendo $74,6 \%$ do gênero feminino, em quatro UBS, do município de Ribeirópolis que tinham o profissional cirurgião-dentista na sua equipe.

As unidades avaliadas foram Centro de Saúde Irmã Alice de Jesus Fernandes, Clínica de Saúde Dr. Dijaume Francisco de Lima, Posto de Saúde Eustáquio de Andrade e Posto de Saúde Gevanda do Nascimento. Do total dos entrevistados, 87,6\% eram da Clínica de Saúde Dr. Dijaume Francisco de Lima, localizada em área urbana, visto que as demais se localizam em áreas rurais.

Ao se avaliar a DAS de acordo com as variáveis socioeconômicas (TABELA 1), verifica-se diferença estatisticamente significativa para os itens gênero e idade, sendo que as mulheres apresentaram maiores níveis de ansiedade do que os homens e a faixa etária de 51 anos ou mais é a menos ansiosa. A maioria da amostra evidenciou uma ansiedade leve (39\%), seguida de muito leve (27.3\%) e moderada (26.6\%), sendo de menor número a ansiedade extrema (7.1\%).

Tabela 1 - “Escala de Ansiedade Dental” (DAS) de acordo com variáveis socioeconômicas

\begin{tabular}{|c|c|c|c|c|c|c|}
\hline & Muito leve & Leve & Moderada & Extrema & Total geral & 2 \\
\hline \multicolumn{7}{|l|}{ Gênero } \\
\hline Feminino & $51(22,2 \%)$ & $\begin{array}{c}87 \\
(37,8 \%)\end{array}$ & $71(30,9 \%)$ & $21(9,1 \%)$ & $230(74,7 \%)$ & 0,0002 \\
\hline Masculino & $33(42,3 \%)$ & $\begin{array}{c}33 \\
(42,3 \%)\end{array}$ & $11(14,1 \%)$ & $1(1,3 \%)$ & $78(25,3 \%)$ & \\
\hline Idade & & & & & & \\
\hline
\end{tabular}




\begin{tabular}{|c|c|c|c|c|c|c|}
\hline & Muito leve & Leve & Moderada & Extrema & Total geral & 2 \\
\hline $18-35$ & $25(19,8 \%)$ & $\begin{array}{c}53 \\
(42 \%)\end{array}$ & $36(28,6 \%)$ & $12(9,5 \%)$ & $126(40,9 \%)$ & 0,01 \\
\hline $36-50$ & $31(28,2 \%)$ & $\begin{array}{c}36 \\
(32,7 \%)\end{array}$ & $36(32,7 \%)$ & $7(6,4 \%)$ & $110(35,7 \%)$ & \\
\hline$\geq \mathbf{5 1}$ & $28(38,9 \%)$ & $\begin{array}{c}31 \\
(43 \%)\end{array}$ & $10(13,9 \%)$ & $3(1,2 \%)$ & $72(23,4 \%)$ & \\
\hline \multicolumn{7}{|c|}{ Nível de instrução } \\
\hline Analfabeto & $10(41,7 \%)$ & $\begin{array}{c}9 \\
(37,5 \%)\end{array}$ & $4(16,7 \%)$ & $1(4,2 \%)$ & $24(7,8 \%)$ & 0,26 \\
\hline Fundamental & $42(26,1 \%)$ & $\begin{array}{c}57 \\
(35,4 \%)\end{array}$ & $50(31,1 \%)$ & $12(7,5 \%)$ & $161(52,3 \%)$ & \\
\hline Médio & $24(42,9 \%)$ & $\begin{array}{c}48 \\
(45,7 \%)\end{array}$ & $24(22,9 \%)$ & $9(8,6 \%)$ & $105(34,1 \%)$ & \\
\hline Superior & $8(44,4 \%)$ & $\begin{array}{c}6 \\
(33,3 \%)\end{array}$ & $4(22,2 \%)$ & $0(0,0 \%)$ & $18(5,8 \%)$ & \\
\hline \multicolumn{7}{|l|}{ Renda } \\
\hline menos de $1 \mathrm{SM}$ & $25(22,1 \%)$ & $\begin{array}{c}38 \\
(33,6 \%)\end{array}$ & $38(33,6 \%)$ & $12(10,6 \%)$ & $113(36,7 \%)$ & 0,11 \\
\hline de 1 a 2 SM & $55(31,3 \%)$ & $\begin{array}{c}74 \\
(42,0 \%)\end{array}$ & $37(21,0 \%)$ & $10(5,7 \%)$ & $176(57,1 \%)$ & \\
\hline de 2 a 3 SM & $3(23,1 \%)$ & $\begin{array}{c}4 \\
(30,8 \%)\end{array}$ & $6(46,2 \%)$ & $0(0,0 \%)$ & $13(4,2 \%)$ & \\
\hline 3 ou mais SM & $1(20,0 \%)$ & $\begin{array}{c}3 \\
(60,0 \%)\end{array}$ & $1(20,0 \%)$ & $0(0,0 \%)$ & $5(1,6 \%)$ & \\
\hline Total (\%) & $84(27,3 \%)$ & $\begin{array}{c}120 \\
(39,0 \%)\end{array}$ & $82(26,6 \%)$ & $22(7,1 \%)$ & $308(100 \%)$ & \\
\hline
\end{tabular}

SM - Salário Mínimo

Fonte: Dados da pesquisa

Foi ainda perguntado aos usuários sobre tempo de espera, sendo que 32,4\% relataram entre 30 minutos a uma hora, $29 \%$ de uma a duas horas, $53 \%$ alegam que neste tempo conversam e $16 \%$ "ficam no celular". Foi ainda perguntado se há alguma palestra no posto e $61 \%$ dos pacientes alegaram que não. Dos 39\% que alegaram ter alguma palestra, $50 \%$ as consideram muito importantes. Quanto à sonoridade $60 \%$ consideram normal ou calmo e quanto a temperatura $68,8 \%$ a consideram adequa- 
da. Sendo 73\% classificaram como boa a avaliação da unidade de saúde. Quanto aos dados socioeconômicos 57\% têm renda de um a dois salários mínimos e 36\% menos que um salário mínimo. Quanto a escolaridade $44.8 \%$ tem ensino fundamental incompleto. Quanto a ocupação $26 \%$ são lavradores, $16 \%$ donas de casa e $9 \%$ aposentados.

Quando os usuários são entrevistados sobre o ambiente e seu estado de ansiedade, a maioria (52\%) respondeu que o ambiente interfere, deixando-os mais ansiosos ou mais calmos. Sendo que $40,3 \%$ consideram o ambiente da sala de espera como algo que os deixa mais ansiosos (FIGURA 2). Ao serem questionados sobre o que poderia amenizar, $27,5 \%$ citaram uma televisão na sala de espera, seguido de $5,8 \%$ de cadeiras mais confortáveis.

Figura 2 - Questionamento sobre a interferência do ambiente da sala de espera na ansiedade. Ribeirópolis, 2019

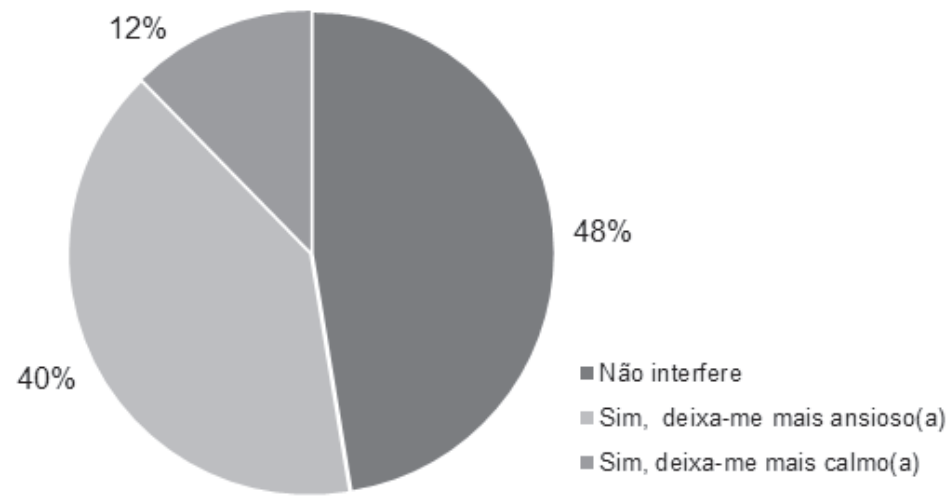

Fonte: Dados da pesquisa

Foi ainda utilizado o PHEQI (FORNARA et al., 2006), para o item conforto físico e espacial (TABELA 2). Sendo demonstrado que os voluntários consideram o ambiente em boa qualidade ou condições, superando $60 \%$ das respostas.

Tabela 2 - Perceived Hospital Environment Quality Indicators (PHEQI), e número total de respostas por item, segundo conforto físico e espacial. Ribeirópolis, 2019

\begin{tabular}{cccccc}
\hline & $\begin{array}{c}\text { Discordo } \\
\text { totalmente } \\
(\mathbf{0})\end{array}$ & $\begin{array}{c}\text { Discordo } \\
\mathbf{( 1 )}\end{array}$ & $\begin{array}{c}\text { Nem discordo } \\
\text { nem concordo } \\
\mathbf{( 2 )}\end{array}$ & $\begin{array}{c}\text { Concordo } \\
\mathbf{( 3 )}\end{array}$ & $\begin{array}{c}\text { Concordo } \\
\text { totalmente } \\
\text { (4) }\end{array}$ \\
\hline $\begin{array}{c}\text { A mobília (armários, cadeiras, } \\
\text { mesas etc.) é de boa qualidade }\end{array}$ & 42 & 7 & 44 & 30 & 185 \\
$\begin{array}{c}\text { As paredes, os pavimentos es os } \\
\text { tetos têm cores bonitas }\end{array}$ & 46 & 6 & 50 & 36 & 168 \\
\hline
\end{tabular}




\begin{tabular}{lccccc}
\hline & $\begin{array}{c}\text { Discordo } \\
\text { totalmente } \\
\text { (0) }\end{array}$ & $\begin{array}{c}\text { Discordo } \\
\mathbf{( 1 )}\end{array}$ & $\begin{array}{c}\text { Nem discordo } \\
\text { nem concordo } \\
\mathbf{( 2 )}\end{array}$ & $\begin{array}{c}\text { Concordo } \\
\text { (3) }\end{array}$ & $\begin{array}{c}\text { Concordo } \\
\text { totalmente } \\
\text { (4) }\end{array}$ \\
\hline $\begin{array}{l}\text { Os lugares sentados (ex.: cadei- } \\
\text { ras, sofás) são pouco cômodos }\end{array}$ & 168 & 21 & 22 & 32 & 65 \\
$\begin{array}{c}\text { As paredes, os pavimentos e os } \\
\text { tetos estão em más condições }\end{array}$ & 204 & 16 & 47 & 15 & 26 \\
$\begin{array}{c}\text { A mobília (cadeiras, mesas etc.) } \\
\text { está em boas condições }\end{array}$ & 28 & 5 & 41 & 30 & 204 \\
$\begin{array}{c}\text { A mobília (cadeiras, mesas etc.) } \\
\text { está em más condições }\end{array}$ & 210 & 24 & 32 & 8 & 34 \\
\hline Fonte: Dados da pesquisa & & & & & \\
\hline
\end{tabular}

Fonte: Dados da pesquisa

Ao se correlacionar a DAS com as questões do PHEQI (TABELA 3) foi observada diferença estatisticamente significativa para todos os índices $(p<0.05)$.

Tabela 3 - Questões de conforto físico e espacial do questionário Perceived Hospital Environment Quality Indicator (PHEQI) correlacionado com o questionário da Escala de Ansiedade Dental (DAS). Ribeirópolis, 2019

\begin{tabular}{cc}
\hline PHEQI & DAS \\
\hline A mobília (armários, cadeiras, mesas etc.) é de boa qualidade & 0.00 \\
As paredes, os pavimentos e os tetos têm cores bonitas & 0.00 \\
Os lugares sentados (ex.: cadeiras, sofás) são pouco cômodos & 0.00 \\
As paredes, os pavimentos e os tetos estão em más condições & 0.02 \\
A mobília (cadeiras, mesas etc.) está em boas condições & 0.00 \\
A mobília (cadeiras, mesas etc.) está em más condições & 0.00 \\
\hline
\end{tabular}

Fonte: Dados da pesquisa

\section{DISCUSSÃO}

O município de Ribeirópolis-SE, localiza-se aproximadamente a 78 km da capital Aracaju, população de 18000 habitantes, sendo em 2017 o rendimento médio de 1.8 salários mínimos (IBGE, 2010). Apresenta um total de doze UBS, sendo oito rurais e quatro urbanas, havendo atendimento odontológico em quatro UBS, sendo destas 1 na área urbana e 2 na área rural (BRASIL, 2017). 
No presente estudo foram entrevistados usuários que frequentam as UBS as quais têm a presença do cirurgião-dentista, sendo a maioria dos entrevistados do gênero feminino (74.67\%). No estudo de Fernandes e colaboradores (2019) realizado em Porto Alegre (RS), verificaram que o gênero feminino foi o mais prevalente em procura pelos cuidados em saúde. Esta relação de maior procura dos serviços pelo gênero feminino é relatada por Schraiber e colaboradores (2010) que para as mulheres há a disciplina do cuidado e para os homens, impropriedades para assistir e cuidar.

Moura e colaboradores (2014) alegam que há lacunas, desde a adequação da estrutura para o atendimento na atenção básica quanto a motivação e desenvolvimento de ações de promoção contra os agravos para a população masculina, o que tem dificultado o seu acesso por serviços de saúde.

A respeito do tempo de espera por atendimento, Oliveira e colaboradores (2019), vinculam o tempo abordado, baseando-se em três aspectos: lista de espera com tempo inadequado às necessidades, tempo de espera incompatível com o atendimento em urgência/emergência e agendamento de serviços em tempo oportuno, sendo considerados ineficientes ao se estudar cinco regiões de saúde no Brasil. No presente estudo a maioria do total de entrevistados alega tempo de espera de 30 minutos a uma hora, considerado curto.

$\mathrm{Na}$ "Escala da Ansiedade Dental", a ansiedade muito leve também pode ser considerada nula desta forma, $72,7 \%$ desta amostra apresenta ansiedade, de leve a extrema, frente ao tratamento odontológico. Percentual, este, inferior aos encontrados em outros estudos, os quais variam entre 95 e 98,3\% (CHAVES et al., 2006; DANTAS, 2014; PEREIRA et al., 2013).

Quanto à prevalência da ansiedade odontológica por gênero não parece haver, entre autores, uma concordância, pois algumas pesquisas sugerem que essa variável não interfere nos resultados (MEDEIROS, 2013; PEREIRA et al., 2013; SANTOS et al., 2007). No entanto, há uma tendência de se obter produtos semelhantes aos aqui encontrados, nos quais o gênero feminino mostra-se mais ansioso (CHAVES et al., 2006; DANTAS, 2014; SINGH et al., 2000), possivelmente, um viés devido ao fato de o homem ser mais conservador e, muitas vezes, sentir dificuldade em exprimir verdadeiramente suas subjetividades (CORAH et al., 1978).

Os pacientes mais velhos de outros estudos revelaram-se mais ansiosos, o que foi explicado como um reflexo do acúmulo de experiências traumáticas ao longo da vida (CARVALHO et al., 2011). Um estudo realizado apenas com mulheres de diferentes faixas etárias, obteve o resultado semelhante de que a ansiedade aumenta com a idade mais avançada (HAGGLIN et al, 1999).

Já outro, contrasta afirmando que pacientes jovens podem ter mais ansiedade por conta de relatos negativos que ouvem de seus pais, avós, entre outros (THOMSON et al., 1997). Semelhante ao nosso estudo, em que a faixa etária de 18 a 35 anos é a mais ansiosa. Em contraponto, pesquisas diferentes não encontraram associação significativa para idade, mas concordam com os resultados deste estudo quanto à ausência de significância estatística sobre renda e nível de instrução (PEREIRA et al., 2013; CHAVES et al., 2006).

Freud (1936) distinguiu a ansiedade em dois tipos, a objetiva, relacionada ao meio ambiente e a neurótica, a qual é exclusivamente psíquica, bem como Corah (1969), que evidencia a sala de espera como um dos fatores que exercem influência sobre a ansiedade dentária. Ao correlacionarmos as 
respostas do $P H E Q /$ com as somas da DAS podemos afirmar, neste estudo, que os pacientes mais ansiosos se importam mais com os aspectos ambientais da sala de espera.

A maioria (52\%) dos entrevistados, seguindo essa linha, acredita que o ambiente da sala de espera interfere na ansiedade, sendo que $40 \%$ acha que ela aumenta e $12 \%$ acha que diminui. Ao perguntarmos como os voluntários desta pesquisa se sentem na sala de espera, $46 \%$ afirmaram ficar relaxados enquanto $37 \%$ se sentem ansiosos. Rodrigues e colaboradores (2015) concluem que uma ambiência acolhedora e humanizada transmite tranquilidade, da mesma maneira que o oposto contribui para aumentar a ansiedade do usuário.

Ao verificar a ambiência e a satisfação dos usuários, encontrou-se que os voluntários consideram o ambiente em boa qualidade ou condições, superando $60 \%$ das respostas. Resultados divergentes foram encontrados no estudo de Cunha e colaboradores (2017) em que os usuários muitas vezes reclamam da falta de materiais e desta forma de resolutividade na atenção. No estudo citado os entrevistados tinham idade mínima de 18 anos e tinham como critério de inclusão no estudo assumisse exercer algum tipo de representatividade (liderança) na comunidade, o que deixa este plano amostral mais crítico (CUNHA et al., 2017). Uma vez que os processos de construção psicossociais e culturais refletem nas descrições, interpretações e avaliações ambientais (BONAIUTO, 2004).

No estudo de Silva e colaboradores (2019), sobre a ambiência do hospital universitário em Minas Gerais, $71,2 \%$ dos participantes responderam que mudariam algo na sala de espera. No presente estudo $59 \%$ consideram os moveis de boa qualidade e $54 \%$ que as paredes têm cores bonitas.

Dos avaliados no presente estudo, $73 \%$ classificaram como boa a avaliação da unidade de saúde, muito provavelmente devido à resolutividade e acesso, apontados como favorável a um cuidado integral e humanizado (CUNHA et al., 2017). A relação positiva entre a expectativa dos usuários e a realidade da saúde pública pode ser explicada com base em seu perfil socioeconômico, em razão de que a maioria não teria condições de pagar por um serviço particular de melhor qualidade (MIALHE et al., 2008).

No presente estudo, $44.5 \%$ dos voluntários tinham mais do que 40 anos, 36.8\% vivem com menos de um salário mínimo, 57.3\% com renda de um a dois salários mínimos, 44.8\% com ensino fundamental incompleto, $26.1 \%$ constituída por lavradores, um público que muitas vezes alegou que não conhecia outro lugar para ter assistência em saúde ou que não conhecia outros municípios longe do local.

Nas salas de espera avaliadas, a necessidade de televisores foi o aspecto de ambiência mais sugerido pelos voluntários, os quais alegam que a carência de entretenimento os faz pensar mais sobre o tratamento o qual serão submetidos, aumentando a ansiedade. De fato, os meios audiovisuais constituem ferramentas aplicáveis à sala de espera e já estão sendo estudados no intuito da redução da ansiedade odontológica (OLIVEIRA, 2009).

Biddiss e colaboradores (2014), em uma revisão de literatura, concluem que, o uso de televisores em salas de espera, embora seja recorrente, não foi suficientemente estudado relacionando-se com a redução da ansiedade, no meio científico, já quanto a musicoterapia, há evidência científica suficiente para provar que se faz eficaz para este fim.

Os usuários aproveitam o tempo ocioso, conversando e trocando saberes. Além disso, demonstram gostar quando um profissional vai à sala de espera acrescentar conhecimento, uma vez que aqueles 
que tiveram a oportunidade de participar de alguma palestra de educação em saúde as consideram muito importantes. Nesse caso, a sala de espera evidencia-se como um ambiente para aprender e conversar (BRONDANI, et al., 2013) o que pode ser melhor explorado, já que a maioria dos entrevistados nunca havia visto uma palestra na sala de espera odontológica em que estavam.

Após 16 anos de PNH, este trabalho salienta a importância da humanização da atenção básica evidenciando seus reflexos sobre a saúde psicossocial do usuário, em foco, aquele a ser submetido a procedimentos odontológicos.

\section{CONCLUSÃO}

Conclui-se que os usuários se sentem longânimes com a ambiência das UBS e consideram o serviço conveniente às suas condições socioeconômicas. Ainda assim, vislumbram a necessidade de melhorias e de acréscimos em confortabilidade. Logo, meios práticos de otimizar a ambiência, como o uso de televisores, devem ser mais estudados e se possível, aplicados.

Nesse contexto, as palestras ministradas pelos profissionais evidenciaram-se importantes meios de humanização, sendo necessária uma maior frequência e acessibilidade destas. Igualmente, a carência ainda presente da inclusão do gênero masculino na atenção e cuidado a saúde, necessitando de políticas de saúde e trabalhistas que os garanta esse direito.

Por fim, reafirma-se que os aspectos físicos, espaciais e humanos compõem um ambiente capaz de interferir positiva ou negativamente na ansiedade de quem o frequenta, fundamentando a notoriedade de uma ambiência harmoniosa em locais de prestação de serviços à saúde.

Sugerimos que estudos futuros apliquem o roteiro de entrevista aqui utilizado em outros nichos amostrais, com diferenças socioeconômicas significantes para que possam se comparar os resultados.

\section{REFERÊNCIAS}

BIDDISSS, E. et al. The effectiveness of interventions aimed at reducing anxiety in health care waiting spaces: a systematic review of randomized and nonrandomized trials. Int. Anesth. Res. Soc., v. 119, n. 2, p. 433-448, 2014.

BRASIL. Ministério da Saúde. Humaniza SUS. Brasilia: MS, 2003 Disponível em: https://www.saude. gov.br/acoes-e-programas/humanizasus. Acesso em: 25 maio 2020.

BRASIL. Ministério da Saúde. Política Nacional de Promoção da Saúde. Brasília: MS, 2006. Disponível em: http://bvsms.saude.gov.br/bvs/publicacoes/politica_promocao_saude.pdf. Acesso em: 31 out. 2019. 
BRASIL. Sistema de Cadastro Nacional de Estabelecimentos de Saúde. 2017 Disponível em: http:// www.cnes.datasus.gov.br/. Acesso em: 21 jan. 2019.

BONAIUTO, M. et al. Indexes of perceived residential environment quality and neighbourhood attachment in urban environments: A confirmation study on the city of Rome. Landscape Urban Plan., v. 65, p. 41-52, 2003.

BONAIUTO, M. Residential satisfaction and perceived residential environment quality. In C. Spielberger ed. Encyclopedia of applied psychology, San Diego: Academic Press, 2004.

BRONDANI, J. E. et al. Percepções de gestantes e puérperas acerca da sala de espera em uma unidade básica de saúde integrada à estratégia saúde da família. Rev. Bras. Prom. Saúde, v. 26, n. 1, p. 63-70,2013.

CARVALHO, R. W. F. et al. Ansiedade frente ao tratamento odontológico: prevalência e fatores predictores em brasileiros. Ciên. Saúde Col., v. 17, n. 7, p. 1915-1922, 2011.

CHAVES, A. M. et al. Estudo epidemiológico da ansiedade dos pacientes ao tratamento odontológico. Rev. Odontol., UNESP, v. 35, n. 4, p. 263-268, 2006.

CORAH, N. L. Development of a dental anxiety scale. J. Dent. Res., v. 48, n. 4, p. 5960, 1969.

CORAH, N. L. et al. Assessment of a dental anxiety scale. J. Am. Dental Assoc., v. 97, p. 816-819, 1978.

CUNHA, A. T. R. et al. Percepções de usuários sobre humanização na Estratégia Saúde da Família: um estudo ancorado na teoria da dádiva. Rev. Ciênc. Plural., v. 3, n. 3, p. 16-31, 2017.

\section{DANTAS, L. P. Efeitos da Passiflora incarnata e do midazolam no controle da ansiedade em} pacientes submetidos à exodontia de terceiros molares inclusos. 2014. 77 f. Dissertação

(Mestrado em Odontologia) - Universidade Federal de Sergipe, Aracaju, 2014.

EMMI, D. T., PIRES, M. J. M. Acolhimento e educação em saúde na sala de espera: Avaliação da contribuição das ações para o atendimento odontopediátrico. Rev. Aten. Saúde, v. 14, n. 48, p. 62-67, 2016.

EMMI, D. T. et al. Humanização no acolhimento aos usuários das clínicas de ensino da Faculdade de Odontologia da Universidade Federal do Pará: cinco anos de experiência de um projeto de extensão. Rev. Conexão, UEPG, v. 12, n. 3, p. 476-486, 2016. 
FERNANDES, L. C. L., BEROLDI, A. D., BARROS, A. D. Utilização de serviço de saúde pela população coberta pela Estratégia de Saúde da Família. Rev. Saúde Públ., v. 43, p. 595-603, 2009.

FORNARA, F., BONAIUTO, M., BONNES, M. Perceived hospital environment quality indicators: A study of orthopaedic units. J. Environ. Psychol., v. 18, n. 26, p. 321-334, 2006.

FREUD, S. The problem of anxiety. New York: The Psychoanalytic Quarterly Press Broadway and W. W. Norton \& Company, 1936.

GARCIA, A. C. P. et al. Análise da organização da Atenção Básica no Espírito Santo: (des)velando cenários. Saúde Debate, v. 38, p. 221-236, 2014.

HAGGLIN, C. et al. Variations in dental anxiety among middleaged and elderly women in Sweden: a longitudinal study between 1968 and 1996.J. Dent. Res., v. 78, n. 10, p. 1655-1661, 1999.

IBGE - Instituto Brasileiro de Geografia e Estatística. Censo Demográfico. 2010. Disponível em: https://cidades.ibge.gov.br/brasil/se/ribeiropolis/panorama. Acesso em: 28 mar. 2019.

MEDEIROS, L. A. Avaliação do grau de ansiedade dos pacientes antes de cirurgias orais menores.

Rev. Odontol., UNESP, v. 42, n. 5, p. 357-363, 2013.

MERHY, E. E. Saúde: a cartografia do trabalho vivo. 2. ed. São Paulo: Hucitec, 2005.

MENDES, E. V. A construção social da atenção primária à saúde. Brasília: CONASS; 2015.

Disponível em: https://www.conass.org.br/biblioteca/pdf/A-CONSTR-SOC-ATEN-PRIM-SAUDE.pdf. Acesso em: 31 out. 2019.

MIALHE, F. L. et al. Avaliação dos usuários sobre a qualidade do serviço odontológico prestado por graduandos do curso de Odontologia da FOP/Unicamp. Rev. Fac. Odont., v. 13, n. 1, p. 19-24, 2008.

MOURA, E. C. et al. Atenção à saúde dos homens no âmbito da Estratégia Saúde da Família. Ciên. Saúde Col., v. 19, n. 2, p. 429-438, 2014.

\section{OLIVEIRA, P. J. P. Influência do espaço do consultório dentário na ansiedade dentária - uma} reflexão 2009. 76 f. Trabalho de Conclusão de Curso (Licenciatura em Medicina Dentária) Universidade Fernando Pessoa, Porto, 2009.

OLIVEIRA, R. A. D. et al. Barreiras de acesso aos serviços em cinco Regiões de Saúde do Brasil: percepção de gestores e profissionais do Sistema Único de Saúde. Cad. Saúde Públ., v. 35, n. 11, p. e00120718, 2019. 
PEREIRA V. Z. et al. Avaliação dos níveis de ansiedade em pacientes submetidos ao tratamento odontológico. Rev. Bras. Ciên. Saúde, v. 17, n. 1, p. 55-64, 2013.

REIS, F. V. et al. Educação em saúde na sala de espera - relato de experiência. Rev. Med. Minas Gerais, v. 24, n. 1, p. 32-36, 2014.

RODRIGUES, M. P. et al. Humanização: fragilidades, desafios e fortalezas em uma escola de odontologia. Rev. Esp. Saúde., v. 16, n. 3, p. 27-38, 2015.

SANTOS, P. A., CAMPOS, J. A. D. B., MARTINS, C. S. Avaliação do sentimento de ansiedade frente ao atendimento odontológico. Rev. Uniara, v. 20, p. 189-201, 2007.

SCHRAIBER, L. B. et al. Necessidades de saúde e masculinidades: atenção primária no cuidado aos homens. Cad Saúde Públ., v. 26, n. 5, p. 961-970, 2010.

SILVA, T. N. R. et al. Sala de espera: uma possibilidade de intervenção em Saúde do Trabalhador. Cad. Bras. Ter. Ocup., v. 27, n. 4, p. 907-916, 2019.

SINGH, K. A. et al. Medo, ansiedade e controle relacionados ao tratamento odontológico. Pesq.

Odont. Bras., v. 14, n. 2, p. 131-136, 2000.

THOMSON, W. M. et al. Changes in self-reported dental anxiety in New Zealand adolescents from ages 15 to 18 years. J. Dent Res., v. 76, n. 6, p. 1287-1291, 1997.

VALENTE, M. A. S. et al. Relato de Experiência - O que te espera na Sala de Espera: educação em saúde em Unidades de Atenção Primária à Saúde (UAPS) de Governador Valadares (MG). Rev. Ext. IFSC, v. 1, n. 3, p. 137-141, 2015. 
1 Acadêmica do Curso de Odontologia, Universidade Federal do Sergipe - UFS. E-mail: lizianemoniq@gmail.com

2 Acadêmico do Curso de Odontologia, Universidade Federal do Sergipe - UFS. E-mail: chaves_moreira@live.com

3 Coordenador de Saúde, Secretaria Municipal de Saúde de Ribeirópolis-SE. E-mail: mcbcabral@hotmail.com

4 Doutora em Odontologia; Professora do Departamento do Curso de Odontologia, Universidade Federal do Sergipe UFS. E-mail: amaralre@yahoo.com.br

\section{(ㅇ) (1) (-)}

Este artigo é licenciado na modalidade acesso abertosob a Atribuição-Compartilhalgual CC BY-SA

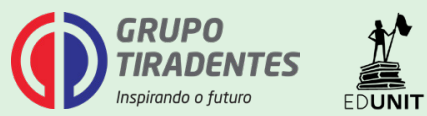

